\title{
Urinary Medium-Chain Acylcarnitines in Medium-Chain Acyl-CoA Dehydrogenase Deficiency, Medium-Chain Triglyceride Feeding and Valproic Acid Therapy: Sensitivity and Specificity of the Radioisotopic Exchange/High Performance Liquid Chromatography Method
}

\author{
EBERHARD SCHMIDT-SOMMERFELD, DUNA PENN, PIERO RINALDO, BRIAN D. KOSSAK, \\ B U. K. LI, ZHI-HENG HUANG, AND DOUGLAS A. GAGE \\ Departments of Pediatrics [E.S., D.P.] and Neurology [B.D.K.], University of Chicago, Pritzker School of \\ Medicine, Chicago, Illinois 60637; Department of Genetics, Yale University School of Medicine, New Haven, \\ Connecticut 06510 [P.R.], Department of Pediatrics, Ohio State University, Columbus, Ohio 43205 [BU.K.L.] \\ and Department of Biochemistry, Michigan State University, East Lansing, Michigan 48824 [Z.H., D.A.G.]
}

\begin{abstract}
To determine the sensitivity and specificity of detecting urinary medium-chain acylcarnitines for the diagnosis of MCAD deficiency, 114 urine specimens from 75 children with metabolic diseases and controls were analyzed in a blinded fashion using a radioisotopic exchange/HPLC method. All 47 patients with MCAD deficiency were correctly diagnosed using the criterion hexanoylcarnitine or octanoylcarnitine peak areas larger than those of other medium-chain acylcarnitines. The majority of them were tested during the asymptomatic state without L-carnitine loading. Four patients with other defects of fatty acid oxidation and three patients receiving valproic acid had a similar acylcarnitine excretion pattern. To further examine the specificity of the method, eight infants receiving a diet enriched with medium-chain triglycerides and 13 additional patients receiving valproic acid were studied. Most of these also tested positive for MCAD deficiency by the above criterion. Analysis by a new gas chromatographic-mass spectrometric procedure revealed that octanoylcarnitine, not valproylcarnitine, was the most abundant medium-chain carnitine ester excreted by a patient treated with valproic acid. Quantitation of urinary hexanoylcarnitine and octanoylcarnitine showed considerable overlap among patients with MCAD deficiency and those receiving valproic acid or a medium-chain triglyceride-enriched diet. MCAD deficiency can be reliably detected in urine specimens by this method without the need for prior carnitine loading. However, other defects in fatty acid oxidation must be differentiated from MCAD deficiency, and a history of medium-chain triglyceride or valproic acid administration must be considered if the diagnosis of MCAD deficiency is sought through analysis of urinary acylcarnitines. (Pediatr Res 31: 545-551, 1992)
\end{abstract}

Received April 2, 1991; accepted January 13, 1992

Correspondence and reprint requests: Eberhard Schmidt-Sommerfeld, M.D. Assistant Professor in Pediatrics, Department of Pediatrics, Box 107. Section of Gastroenterology, Hepatology and Nutrition, University of Chicago, 5825 S. Maryland Ave., Chicago, IL 60637.

Supported in part by grants from the Biotechnology Resource Branch, National Center for Research Resources, NIH (RR-00480) (Z.H., D.A.G.); the Clinical Nutritional Research Unit, University of Chicago (NIDDK 5P30DK26678-12) (D.P); the Block Fund for Basic Research and Advanced Study (E.S.); the Home Health Care Fund (E.S.); and the Joseph Drown Foundation (P.R.).

\section{Abbreviations}

MCAD, medium-chain acyl-CoA dehydrogenase C6, hexanoylcarnitine

C8, octanoylcarnitine

MCT, medium-chain triglyceride

GC/MS, gas chromatography/mass spectrometry

FAB/MS, fast atom bombardment/mass spectrometry

MC, medium-chain acylcarnitine

r.t., retention time

DC, decanoylcarnitine

MCAD deficiency is the most common inborn error of fatty acid oxidation (for review see Ref. 1). It usually presents during the first 2 y of life as an acute episode of lethargy, coma, or unexpected death after a period of fasting, often associated with infection. Typical laboratory findings include hypoketotic hypoglycemia and dicarboxylic aciduria. A fatty liver is found on biopsy or at autopsy. The disease is characterized by sudden, lifethreatening metabolic crises carrying a high mortality rate, but the prognosis is excellent if such crises are successfully overcome or prevented by avoidance of fasting. Therefore, early diagnosis before a life-threatening event occurs is warranted.

However, the diagnosis may be missed by routine GC/MS of urinary organic acids in the asymptomatic state unless a potentially dangerous fasting test is performed (1). Besides enzymatic $(2,3)$ and molecular $(4)$ analysis, two established biochemical methods are frequently used to diagnose MCAD deficiency: quantitation of urinary acylglycines by stable isotope dilution (5) and detection of MC by FAB/MS (6). The sensitivity of the latter method for the diagnosis of MCAD deficiency from urine in the absence of $L$-carnitine loading has been questioned $(7,8)$. Unlike acylglycines, acylcarnitines may be excreted in very small quantities by patients with MCAD deficiency who are carnitine deficient and, therefore, may not be detected by FAB/MS. Recently, the use of fast atom bombardment/tandem mass spectrometry has been shown to be more sensitive (9). However, even with this methodologic improvement, it is recognized that the interpretation of acylcarnitine profiles is more reliable if the urine is collected after the administration of a carnitine bolus (9).

The objective of the present study was to determine whether a 
different methodologic approach to the detection of urinary carnitine esters characteristic of MCAD deficiency can overcome these problems and facilitate the diagnosis in the asymptomatic state without L-carnitine loading. To our knowledge, this is the first blinded study testing the sensitivity and specificity of the detection of urinary MC to diagnose MCAD deficiency.

\section{MATERIALS AND METHODS}

Blinded analysis. One hundred fourteen coded urine specimens from 75 children were sent on dry ice from the Biochemical Disease Detection Laboratory of the Department of Genetics at Yale University to the Division of Gastroenterology, Hepatology, and Nutrition of the Department of Pediatrics, University of Chicago, for carnitine ester analysis. The specimens had been kept frozen at $-20^{\circ} \mathrm{C}$ for up to $2 \mathrm{y}$. No clinical or laboratory data were provided at the time of analysis and evaluation of the data. The code was broken after the results had been reported by the analyzing laboratory.

Based on previous experience with the radioisotopic exchange/ HPLC method (10), the criterion for making the diagnosis of MCAD deficiency was set a priori as follows: positive test $=\mathrm{C} 6$ or $\mathrm{C} 8$ peak areas larger than those of other MC; negative test $=$ C6 or C8 peak areas smaller than those of other MC. The other $\mathrm{MC}$ were defined as compounds with a retention time $>28 \mathrm{~min}$ and distinct from $\mathrm{C} 6$ and $\mathrm{C} 8$ on HPLC. Some have previously been identified in normal human urine $(11-13)$.

Family studies. Urine specimens were obtained from all members of five families who had at least one child with MCAD deficiency. In five children of three families, the diagnosis of MCAD deficiency had already been made, and in five siblings this diagnosis had already been excluded by carnitine ester analysis with FAB/MS after carnitine loading (Drs. D. S. Millington and C. R. Roe, Duke University).

In the other two families, MCAD deficiency was first detected by acylcarnitine analysis with the radioisotopic exchange/HPLC method in two children and tentatively excluded in three siblings. One of these families had lost a child at 8 mo of age during an acute episode suggestive of MCAD deficiency. A fatty liver was found at autopsy, but no material was available for retrospective analysis. In one of the two affected children, the diagnosis was confirmed by enzyme measurements (Drs. D. E. Hale and P. M. Coates, Children's Hospital of Philadelphia) and DNA analysis (Drs. I. Yokota and K. Tanaka, Yale University) in skin fibroblasts. In the other affected child, the diagnosis was confirmed by DNA analysis of a blood spot specimen (Dr. Y. Matsubara, Tohoku University). The three siblings were confirmed by urinary acylglycine analysis to be unaffected (5).

$M C T$ feeding or valproic acid therapy. To further investigate the specificity of the method, urine specimens were collected from eight infants (2-11 mo of age) with gastrointestinal diseases who received a formula containing either $40 \%$ [Pregestimil (Mead Johnson, Evansville, IN), $n=3$ ] or $85 \%$ [Portagen (Mead Johnson), $n=5$ ] of the fat as MCT. The formulas were not supplemented with L-carnitine. In addition, urine specimens were collected from 13 children (4-15 y of age) who received valproic acid for seizure control. In four of them, a urine sample was collected before valproic acid therapy was started.

Radioisotopic exchange HPLC. Urinary free and total carnitine were determined radiochemically as previously described (12). For the separation of individual carnitine esters, a radioisotopic exchange assay was performed according to Bieber and Kerner (14) with the following modifications: Twenty $\mu \mathrm{L}$ of native or diluted urine (containing $0.5-1.0 \mathrm{nmol}$ total carnitine) were used for the incubation. Extraction and purification procedures before the exchange assay were found to be unnecessary for the purpose of this study and were therefore omitted. Later in the course of the study, the nine urine specimens with the highest total carnitine content (up to $3.6 \mathrm{mmol} / \mathrm{L}$ ) were reanalyzed without prior dilution. The results were not different from those of the dilute specimens indicating that, for the detection of medium-chain carnitine esters, dilution of the urine was unnecessary even with high total carnitine concentrations. The reaction mixture contained the same components as previously described (14), except that the amount of dialyzed carnitine acetyl transferase (Boehringer, Mannheim, Germany) was increased to $4 \mathrm{U}$. The incubation period was extended to $2 \mathrm{~h}$ at $20^{\circ} \mathrm{C}$. Subsequently, $10 \mu \mathrm{L}$ of $30 \mathrm{mmol} / \mathrm{L} \mathrm{N}$-ethylmaleimide were added and the incubation was continued for 15 additional min. To avoid precipitation of medium-chain carnitine esters, the sample was treated with $50 \mu \mathrm{L}$ of methanol instead of perchloric acid, placed on ice for $10 \mathrm{~min}$, centrifuged, and filtered (14). Seventy $\mu \mathrm{L}$ of filtrate were injected for HPLC separation (Waters, Milford, MA) using a $\mathrm{C} 18$ reverse phase column (Waters $\mu$-Bondapak). The mobile phase consisted of $5 \mathrm{mmol} / \mathrm{L}$ aqueous butane sulfonic acid, $5 \mathrm{mmol} / \mathrm{L}$ ammonium acetate, adjusted to $\mathrm{pH} 3.4$ with glacial acetic acid (solvent A), and $100 \%$ methanol (solvent B). Flow rate and developing program were as described by Kerner and Bieber (15). The radioactivity in the effluent was detected by a $\beta$-radioactive flow detector (FLO-ONE model CR; Radiomatic Instruments, Tampa, FL), recorded and integrated by a computer.

A mixture of authentic standards containing L-acetylcarnitine, L-propionylcarnitine, D, L-butyrylcarnitine, L-isovalerylcarnitine, L-C6, L-C8, and L-DC (Sigma Chemical Co., St. Louis, MO, or synthesized according to Ref. 16) was incubated in the same manner as the urine and injected at the beginning and the end of each series of approximately 10 urine samples. This allowed monitoring of appropriate radioisotopic exchange of ${ }^{3} \mathrm{H}$-carnitine into the acylcarnitine pool and identification of sample peaks by comparing their retention times with those of the respective standards.

Quantitation of $C 6$ and $C 8$. The validity of the radioisotopic exchange/HPLC method for the quantitation of C6 and C8 in urine was tested as follows: To $1 \mathrm{~mL}$ of dilute urine from a control subject containing approximately $30 \mathrm{nmol}$ total carnitine were added 0.5 or $5 \mathrm{nmol}$ each of authentic L-C6 and L-C8. These two concentrations are representative of the concentrations of $\mathrm{C} 6$ and $\mathrm{C} 8$ found in the urine of asymptomatic patients with MCAD deficiency without or with carnitine therapy, respectively (10). The exact concentration of each added standard was determined by the median value of six radiochemical measurements of acylcarnitine (12). These values represent the expected values given in Table 1 . The radioisotopic exchange/ HPLC method was performed with native urine and also five times each with urine containing the lower and higher concentrations of standards. C6 and C8 were quantitated on the basis of the specific radioactivity in the free carnitine peak (14). The values were corrected for minor concentrations of C6 and C8 found in the native urine. Means and ranges of the measured values approximated $100 \%$ of the expected values for C6 in both concentration ranges and for $\mathrm{C} 8$ in the lower concentration range. With the higher concentration of $\mathrm{C} 8$, the mean measured value was $89 \%$ of the expected value.

C6 and C8 were quantitated in randomly selected urine sam-

Table 1. Recovery of C6 and C8 standards added to urine specimen*

\begin{tabular}{|c|c|c|c|c|c|}
\hline \multicolumn{3}{|c|}{$\mathrm{C} 6(\mu \mathrm{mol} / \mathrm{L})$} & \multicolumn{3}{|c|}{$\mathrm{C} 8(\mu \mathrm{mol} / \mathrm{L})$} \\
\hline Measured & Expected & $\%$ & Measured & Expected & $\%$ \\
\hline $\begin{array}{c}0.51 \\
(0.40-0.53)\end{array}$ & 0.50 & 102 & $\begin{array}{c}0.49 \\
(0.41-0.55)\end{array}$ & 0.48 & 98 \\
\hline $\begin{array}{c}3.69 \\
(3.65-3.72)\end{array}$ & 3.68 & 100 & $\begin{array}{c}3.16 \\
(3.08-3.20)\end{array}$ & 3.56 & 89 \\
\hline
\end{tabular}

* The expected values were determined by the median of six repeated measurements (radiochemical method). The measured values represent the mean (range) from five radioisotopic exchange/HPLC procedures. For details, see text. 
ples from 12 asymptomatic patients with MCAD deficiency (six were treated with L-carnitine at the time of urine collection), six patients treated with valproic acid, and six infants receiving a formula enriched with MCT. Before the radioisotopic exchange reaction, known amounts of $\mathrm{L}-\mathrm{DC}$ were added to appropriately diluted urine samples (total carnitine $25-50 \mu \mathrm{mol} / \mathrm{L}$ ) as internal standard. The exact concentration of $\mathrm{DC}$ was determined radiochemically (12). To those samples that contained little free carnitine ( $<30 \%$ of total), a known amount of free carnitine was also added to assure complete radioisotopic exchange. Amounts of $\mathrm{C} 6$ and $\mathrm{C} 8$ were calculated in two ways (14):

$$
\begin{aligned}
& \left.\mathrm{nmol} \mathrm{AC}=\frac{\mathrm{dpm} \text { in } \mathrm{AC}}{\mathrm{dpm} \text { in } \mathrm{FC}} \times \mathrm{nmol} \mathrm{FC} \quad \text { (value } \mathrm{A}\right) \\
& \left.\mathrm{nmol} \mathrm{AC}=\frac{\mathrm{dpm} \text { in } \mathrm{AC}}{\mathrm{dpm} \text { in } \mathrm{DC}} \times \mathrm{nmol} \mathrm{DC} \quad \text { (value } \mathrm{B}\right)
\end{aligned}
$$

where $\mathrm{AC}$ is the calculated value for the acylcarnitine (C6 and $\mathrm{C} 8$ ) in question and FC and DC are the known amounts of free carnitine and decanoylcarnitine, respectively.

There was good agreement between these two calculations of C8 (value A versus value B, $r=0.997$ by linear regression analysis, Fig. 1) as well as of $\mathrm{C} 6(r=0.993)$, suggesting that radioisotopic equilibrium had been reached for $\mathrm{C} 6, \mathrm{C} 8$, and DC in the urine samples measured.

Identification of medium-chain carnitine esters. A urine specimen $(2 \mathrm{~mL})$ from a patient with MCAD deficiency treated with L-carnitine was extracted twice with $19 \mathrm{vol}$ chloroform/methanol $(3: 2, \mathrm{vol} / \mathrm{vol})$ and dried under a stream of nitrogen. For marker purposes, the isotopic exchange reaction was performed with a $20-\mu \mathrm{L}$ aliquot of the native urine. The dried samples were dissolved in methanol, added to the labeled aliquot, applied in five portions to the HPLC column, and eluted as described above. The labeled acylcarnitines in question were repeatedly collected from the column by fraction collection, pooled, dried, and subsequently analyzed by two different GC/MS techniques: 1 ) as the corresponding acyl- $\mathrm{N}$-demethylcarnitine propyl-esters [The acylcarnitines were derivatized by a two-step procedure that involved a mild esterification using $n$-propyl chloroformate in aqueous $n$-PrOH-pyridine followed by an on-column dequaternization in the presence of iodide during GC-chemical ionization

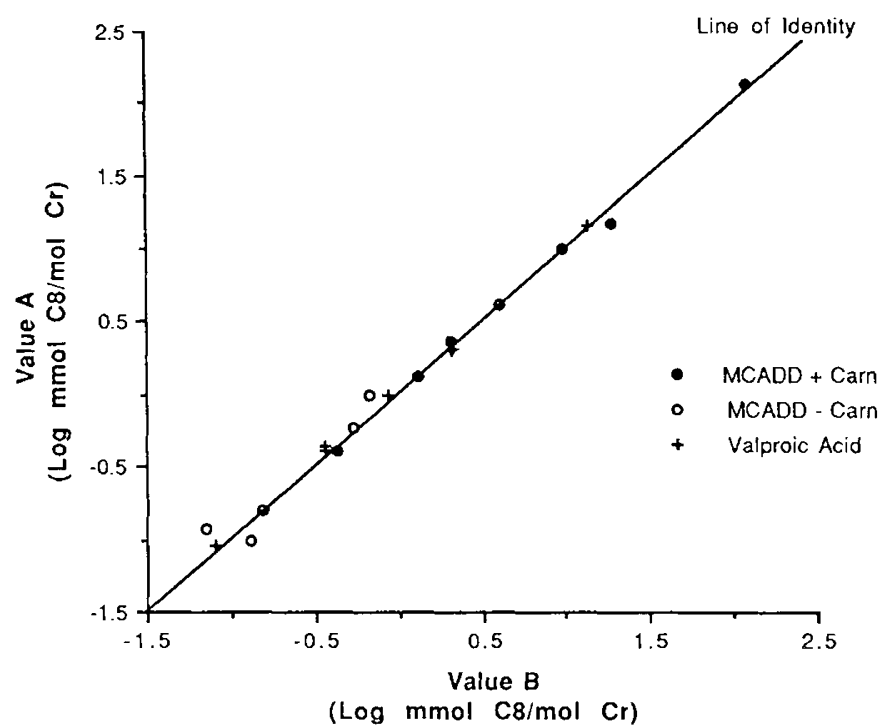

Fig. 1. Comparison of two methods of calculating the concentration of $\mathrm{C} 8$ in urine samples from patients with MCAD deficiency $(M C A D D)$ with $(+$ Carn; $n=6)$ and without $(-\operatorname{Carn} ; n=6)$ L-carnitine therapy and from those receiving valproic acid $(n=6)$. Value $A$ is based on the specific radioactivity of free carnitine; value $B$ is based on the specific radioactivity of the DC standard. The line of identity is shown.
(methane)-MS (17).], and 2) as the trimethylsilyl-derivatives of the acyl moieties after alkaline hydrolysis (analysis performed by Dr. M. J. Bennett, Children's Hospital of Philadelphia).

In addition, acylcarnitines in a urine specimen from a patient treated with valproic acid were purified and enriched by the use of a microcolumn of $\mathrm{AG} 1 \times 8$ anion exchange resin (Bio-Rad Laboratories, Richmond, CA) and a $\mathrm{Cl} 8$ cartridge (Baxter Healthcare Corp., Muskegan, WI) as described by Millington et al. (9) and subsequently analyzed as their acyl-N-demethylcarnitine propyl esters as described above (17).

\section{RESULTS}

Blinded study. In the blinded analysis of 114 urine specimens, 87 specimens from 54 subjects tested positive according to the diagnostic criterion, i.e. the $\mathrm{C} 8$ peak area was larger than the peak areas of other MC. Furthermore, the $\mathrm{C} 8$ peak area was larger than that of C6 (Fig. $2 a$ ). Seventy-nine of these specimens were from 47 patients with MCAD deficiency (Table 2): Four patients lived in Australia, two in Europe, and the rest in 18 cities around the United States. All showed abnormal urinary acylglycine concentrations (5). Acylcarnitines had been analyzed by $\mathrm{FAB} / \mathrm{MS}$ in 19 cases, but the diagnosis had initially been missed in four cases with this method (7). In the majority of cases, the diagnosis was confirmed by enzymatic assays $(2,3)$ and in 27 cases by DNA analysis (4): 23 patients were found to be homozygous for the prevalent ${ }^{985} \mathrm{~A}-\mathrm{G}$ mutation, three patients carried a rare second mutation, and one patient was found to be a compound heterozygote for two rare mutations (18). Sixty-six of the 79 urine specimens from MCAD-deficient patients were obtained during the asymptomatic state without treatment with L-carnitine.

Of the 87 "positive" specimens, two were from a patient with ethylmalonic-adipic aciduria and one was from a patient with evidence of a defect in branched-chain acyl-CoA oxidation (19). In these three specimens, $\mathrm{C} 6$ and $\mathrm{C} 8$ peaks were present, but, in the former patient, butyrylcarnitine and, in the latter patient, isobutyrylcarnitine were the major carnitine esters excreted. Two specimens were from patients with glutaric aciduria type II who were both treated with $\mathrm{L}$-carnitine. In these, $\mathrm{C} 6$ and $\mathrm{C} 8$ peaks were small. Three specimens with a similar carnitine ester excretion pattern were from children treated with valproic acid for a seizure disorder.

Twenty-seven urine specimens from 21 subjects tested negative (C6 and C8 < MC, Table 2). Seven of these were from two asymptomatic individuals examined during family screening who were heterozygous for the ${ }^{985} \mathrm{~A}-\mathrm{G}$ mutation of the MCAD gene. Hexanoylglycine was present in their urine. Enzyme assays by electron transfer flavoprotein-reduction (2) and ${ }^{3} \mathrm{H}$-release (3) in cultured fibroblasts, however, yielded conflicting results (20). Radioisotopic exchange/HPLC showed the presence of small amounts of $\mathrm{C} 6$ and $\mathrm{C} 8$ in urine, but the set criterion for MCAD deficiency was not fulfilled. No abnormal carnitine esters were detected by FAB/MS (analysis performed by Dr. C. R. Roe, Duke University). The remaining specimens were from children with other metabolic diseases and from normal controls (Table 2 , Fig. $2 b$ ). No urine specimen from a patient with the unequivocal diagnosis of MCAD deficiency tested negative (100\% sensitivity).

Family study. In the five families studied, all children with MCAD deficiency were correctly diagnosed. The parents and all nonaffected siblings tested negative (Table 3 ).

$M C T$-feeding, valproic acid therapy, and normal controls. In the urine of all eight infants who received MCT in their diet, peak areas of $\mathrm{C} 6$ and $\mathrm{C} 8$ were larger than those of other MC (Fig. $3 a$, Table 4)

In the urine of nine out of 13 children receiving valproic acid, peak areas of C6 and C8 were larger than those of other MC (Fig. 3b, Table 4). Valproylcarnitine was not detected, presumably because of a lack of substrate specificity for the enzyme 

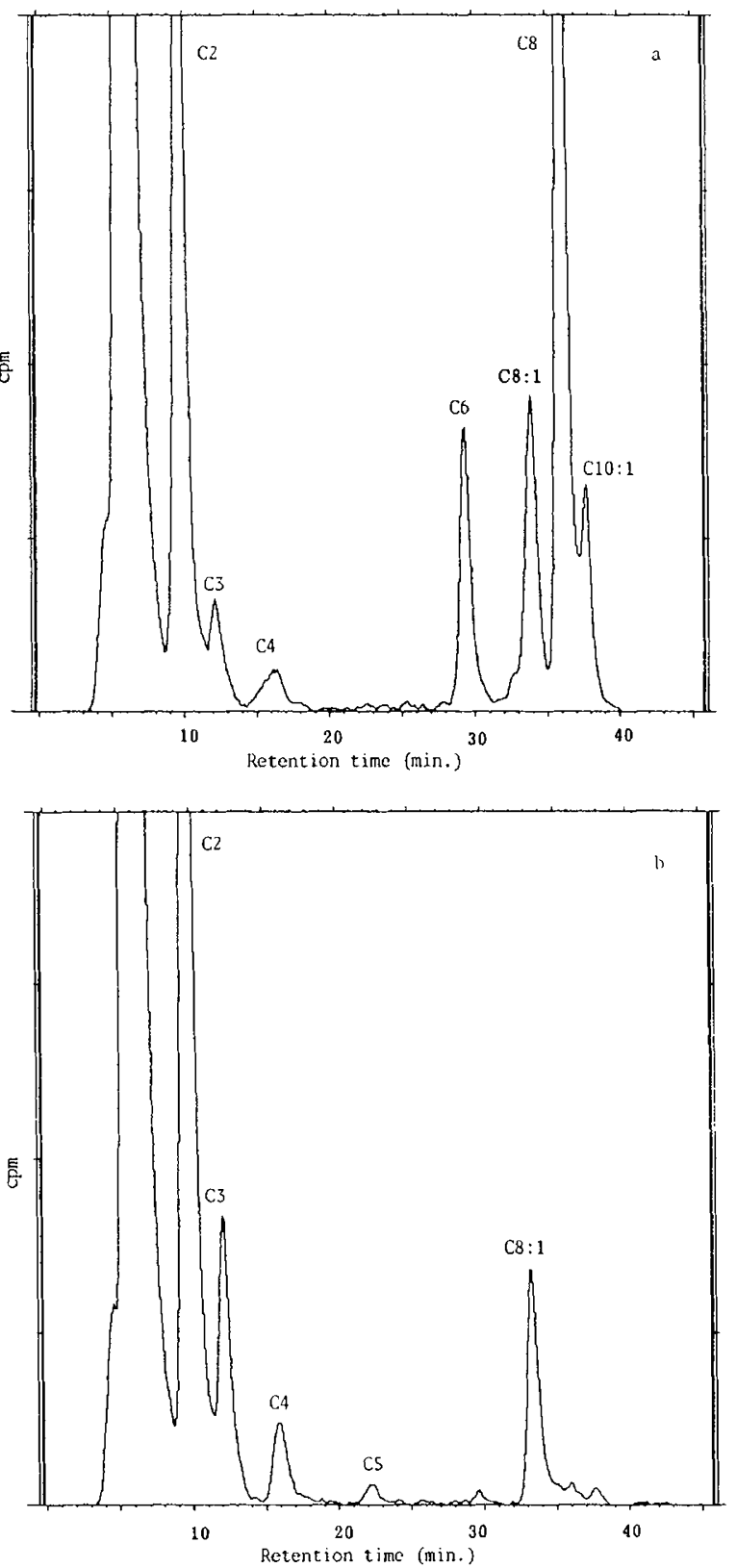

Fig. 2. HPLC profiles of urinary carnitine esters of $(a)$ a 2 -y-old patient with MCAD deficiency and $(b)$ a 10-y-old control child. $C 2$, acetylcarnitine; $C 3$, propionylcarnitine; $C 4$, (iso)-butyrylcarnitine; $C 5$, C5-acylcarnitine; $C 8: 1$, octenoylcarnitine; and $C 10: 1$, decenoylcarnitine. The retention time of C8:1 is slightly different in $a v s b$, indicating structurally different metabolites (see text).

carnitine acetyl transferase used in the assay (Bieber LL, personal communication). GC/MS analysis of the acylcarnitines in a representative urine sample from a valproic acid-treated patient showed the presence of three saturated eight-carbon acylcarnitines (Fig. $4 a$ ): The predominant compound was C8. Smaller amounts of valproylcarnitine and another $\mathrm{C} 8: 0$-acylcarnitine isomer were also detected. The mass spectrum of the derivatized $\mathrm{C} 8$ is shown in Fig. $4 b$. Urine specimens from this patient and from three additional patients were collected before initiation of valproic acid therapy. In none of them were C6 and C8 peaks larger than those of other MC.

Sixteen urine samples obtained from normal control children occasionally exhibited minor $\mathrm{C} 6$ and $\mathrm{C} 8$ peaks, which were always smaller than peaks of other MC (primarily octenoylcarnitine) consistently present (Fig. $2 b$ ).

Quantitation of C6 and C8. The concentrations of $\mathrm{C6}$ and $\mathrm{C} 8$ in the urine of patients with MCAD deficiency not receiving Lcarnitine were in the same range as those of patients treated with valproic acid or of infants receiving MCT-enriched formulas (Table 5). Their median value was higher in MCAD-deficient patients receiving L-carnitine therapy, but the concentration range was wide and considerable overlap was noted among all groups (Fig. 1). If minor peaks of $\mathrm{C} 6$ and $\mathrm{C} 8$ were detected in normal control children, they were poorly separated from other MC, making quantitation unreliable.

Minor medium-chain carnitine esters. Two additional medium-chain carnitine esters were detected in the urine of patients with MCAD deficiency. One had an r.t. shorter than that of $\mathrm{C} 8$ (mean $\Delta$ r.t. from C8: -1.7 min; Fig. $2 a$ ) but longer than that of the predominant medium-chain carnitine ester found in the urine of control children (mean $\Delta$ r.t. from C8: -2.7 min; Fig. $2 b$ ). Mixing urines from a patient with MCAD deficiency and from a control child resulted in detection of a double peak in the area in question, showing that these metabolites are structurally distinct. The former metabolite (mean $\Delta$ r.t. from C8: $-1.7 \mathrm{~min}$ ) was present in all urine specimens from patients with MCAD deficiency and was identified as an octenoylcarnitine by both GC/MS methods used. It was not detected by HPLC in the urine of controls, children receiving MCT or valproic acid, and patients with glutaric aciduria type II. The latter metabolite (mean $\Delta$ r.t. from C8: $-2.7 \mathrm{~min}$ ) was always present in the urine of normal controls and has previously been shown to be $\Delta 6$-octenoylcarnitine (11). It was also found in the urine of children receiving MCT or valproic acid (Fig. 3) and patients with glutaric aciduria type II. The nature of the structural difference between these metabolites is unknown at present, but may be related to the position of the double bond or cis-trans configuration.

The other additional medium-chain carnitine ester detected in $75 \%$ of the MCAD-deficient patients had an r.t. longer than that of $\mathrm{C} 8$ (mean $\Delta$ r.t. from C8: +1.5 min; Fig. $2 a$ ). This metabolite was identified as a decenoylcarnitine by both GC/MS procedures. In $25 \%$ of the patients with MCAD deficiency, it was not clearly separated from the C8 peak by HPLC. It appeared to be present in the urine of most patients treated with valproic acid (Fig. $3 b$ ) and occasionally in control urines, but was not clearly identified in the urine of infants fed MCT.

\section{DISCUSSION}

This study confirms the presence in the urine of patients with MCAD deficiency of characteristic MC that have previously been detected using different techniques $(6,8,23-27)$. However, the sensitivity and specificity of these methods for the diagnosis of this disease have never been tested. The results of our blinded analysis of urine suggest that MCAD deficiency can be reliably detected by the radioisotopic exchange/HPLC method even if urinary acylcarnitine concentrations are very low, i.e. in the asymptomatic state without fasting or $\mathrm{L}$-carnitine loading. The high sensitivity of the method is achieved by 1 ) use of the enzyme carnitine acetyl transferase which eliminates "background noise" caused by compounds other than carnitine esters, and 2) use of a radioisotope tracer. The lower limit of detection of individual carnitine esters in urine was estimated to be $0.01 \mu \mathrm{mol} / \mathrm{L}$. The sensitivity of the method is less dependent on the absolute amounts of carnitine esters present than on their concentration relative to free carnitine. Therefore, the method favors the detection of carnitine esters in the urine of patients with secondary carnitine deficiency, where the free carnitine concentration is low relative to acylcarnitine. In this situation, most of the radioactivity is exchanged into the acylcarnitine pool. In fact, the sensitivity of detection of carnitine esters decreases if free carnitine is added to the urine specimen (unpublished data).

In addition, small concentrations (at least $0.5 \mu \mathrm{mol} / \mathrm{L}$ ) of $\mathrm{C} 6$ and $\mathrm{C} 8$ can be quantitated provided that radioisotopic equilibrium is reached during the exchange reaction. This was demonstrated in two ways: 1 ) by adding different amounts of $\mathrm{C} 6$ and 
Table 2. Blinded analysis of 114 urine specimens from 75 subjects*

\begin{tabular}{|c|c|c|c|c|}
\hline HPLC pattern & Diagnosis & Established by & $\begin{array}{c}\text { Specimens } \\
(n)\end{array}$ & $\begin{array}{c}\text { Subjects } \\
(n)\end{array}$ \\
\hline \multirow[t]{13}{*}{$\mathrm{C} 6, \mathrm{C} 8>\mathrm{MC}$ (positive) } & MCADD & Acylglycines & 79 & 47 \\
\hline & & Acylcarnitines (FAB/MS) & 21 & 19 \\
\hline & & Enzyme analysis & & 34 \\
\hline & & ETF reduction & & 32 \\
\hline & & ${ }^{3} \mathrm{H}$ release & & 2 \\
\hline & & Molecular analysis & & 27 \\
\hline & EMAA & Acylglycines & 2 & 1 \\
\hline & & ETF-QO & & 1 \\
\hline & GA II & Organic acids & 2 & 2 \\
\hline & & Acylglycines & 2 & 2 \\
\hline & BCAO defect (?) & Organic acids & 1 & 1 \\
\hline & & Acylglycines & 1 & 1 \\
\hline & Valproic acid & History & 3 & 3 \\
\hline \multirow[t]{8}{*}{$\mathrm{C} 6, \mathrm{C} 8<\mathrm{MC}$ (negative) } & Heterozygote & Molecular analysis & & 2 \\
\hline & MCADD & Enzyme analysis & & 2 \\
\hline & SCADD & Enzyme analysis & & 2 \\
\hline & GA II & Organic acids & 3 & 2 \\
\hline & & Acylglycines & 3 & 2 \\
\hline & IEM, undefined & Organic acids & 1 & 1 \\
\hline & MCADD excluded & Acylglycines & 1 & 1 \\
\hline & Normal controls & & 14 & 14 \\
\hline
\end{tabular}

* MCADD, MCAD deficiency; MC, other medium-chain acylcarnitines; EMAA, ethylmalonic adipic aciduria; GA II, glutaric aciduria type II; BCAO, branched-chain acyl-CoA oxidation (20); SCADD, short-chain acyl-CoA dehydrogenase deficiency; IEM, inborn error of metabolism; and ETF-QO, electron transfer flavoprotein ubiquinone oxidoreductase $(31,32)$.

Table 3. Carnitine ester analysis in urine specimens from five families affected by MCAD deficiency*

\begin{tabular}{lrcc}
\multicolumn{4}{c}{ families affected by $M C A D$ deficiency* } \\
& $n$ & $\begin{array}{c}\mathrm{C} 6, \mathrm{C} 8>\mathrm{MC} \\
\text { (positive) }\end{array}$ & $\begin{array}{c}\mathrm{C} 6, \mathrm{C} 8<\mathrm{MC} \\
\text { (negative) }\end{array}$ \\
\hline Affected children & 7 & 7 & 0 \\
Parents & 10 & 0 & 10 \\
Nonaffected siblings $\dagger$ & 8 & 0 & 8 \\
\hline
\end{tabular}

* Patients from the blinded analysis (Table 2) are not included.

$\dagger$ May include heterozygotes.

C8 standards to a control urine specimen, and 2) by calculating the concentrations of $\mathrm{C} 6$ and $\mathrm{C} 8$ in patients' urine samples on the basis of both the specific radioactivity of free carnitine and that of the internal standard DC. Both calculations yielded similar results, suggesting that isotopic equilibrium was reached for $\mathrm{C} 6, \mathrm{C} 8$, and DC in the urine specimens analyzed.

Although the sensitivity of the method is high, our results cast doubt on the notion that the excretion of certain medium-chain carnitine esters in urine is specific for MCAD deficiency (1).

First, C6 and C8 as well as decenoylcarnitine were occasionally found in the urine of controls and $\Delta 6$-octenoylcarnitine has previously been shown to be the major medium-chain carnitine ester excreted by normal individuals $(11,13)$.

Second, C6, C8, and octenoylcarnitine were almost invariably present in the urine collected from infants receiving MCT. The physiologic significance of this phenomenon is unclear. It may indicate an overloading of the $\beta$-oxidation pathway and an accumulation of medium-chain acyl-CoA during MCT feeding. This hypothesis is supported by the appearance of $\mathrm{C} 6$ and $\mathrm{C} 8$ in the plasma, urine, bile, and liver tissue of newborn piglets after enteral or parenteral administration of MCT (28). In contrast to MCAD deficiency, in which most of the MC are thought to originate in mitochondria, the formation of these metabolites during MCT administration may also occur in the cytosol through microsomal carnitine acyl transferases with a specificity for medium-chain acyl-CoA (21). Quantitation of C6 and C8 shows that, during MCT feeding, the excretion of C6 is similar to that of $\mathrm{C} 8$. This is in contrast to patients with MCAD deficiency, in whom $\mathrm{C} 8$ is always the more predominant medium-chain carnitine ester excreted.

Third, C6, C8, octenoylcarnitine, and decenoylcarnitine are present in the urine of most patients treated with valproic acid. The amounts are in the same range as those excreted in MCAD deficiency. Millington et al. (22) reported the excretion of valproylcarnitine in the urine of patients treated with valproic acid. They also found traces of its isomer, C8. In contrast, our GC/ MS analysis revealed $\mathrm{C} 8$ as the prominent saturated eight-carbon acylcarnitine isomer, with a much smaller quantity of valproylcarnitine detected. This discrepancy with the literature may be related to methodologic differences or individual variations in the acylcarnitine excretion pattern of valproic acid-treated patients. Our findings suggest that valproic acid treatment may result in an inhibition of fatty acid oxidation partly at the level of MCAD and are in agreement with the recent finding of a moderate inhibitory effect of valproyl-CoA on human liver MCAD in vitro (29). However, they do not support the concept that conjugation of valproic acid with carnitine is an important "detoxifying" mechanism in the human.

Patients with MCAD deficiency consistently excreted two additional carnitine esters: octenoylcarnitine and decenoylcarnitine. These metabolites have previously been identified by FAB/ MS (9). However, in our HPLC system, the octenoylcarnitine present in the urine of patients with MCAD deficiency had a retention time distinct from that of the $\Delta 6$-octenoylcarnitine found in the urine of metabolically normal individuals, demonstrating structural differences between these two metabolites. The octenoylcarnitine with the retention time closer to that of $\mathrm{C} 8$ may be more specific for MCAD deficiency.

The results of our family study confirm the previous observation (30) that carriers of MCAD deficiency cannot be identified by urinary acylcarnitine analysis.

In conclusion, using the radioisotopic exchange/HPLC method in blinded analysis of acylcarnitines in urine, we were 

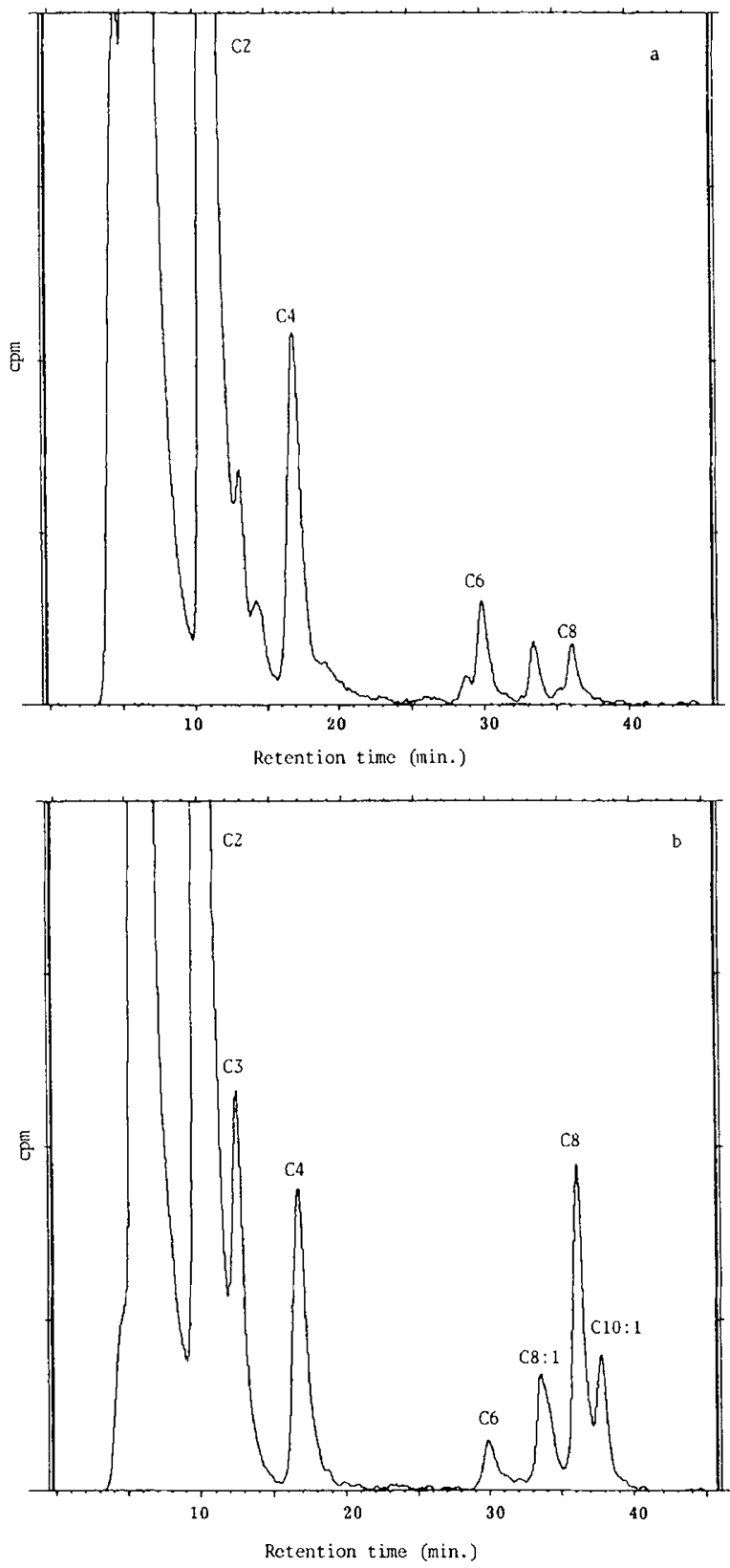

Fig. 3. HPLC profiles of urinary carnitine esters of $(a)$ a 3-mo-old metabolically normal infant receiving a formula (Portagen) supplemented with MCT and $(b)$ a 15 -y-old patient without known metabolic disease receiving valproic acid for seizures. No significant peaks of $\mathrm{C} 6$, $\mathrm{C} 8$, or decenoylcarnitine $(\mathrm{CIO}: 1)$ were found before initiation of valproic acid treatment. $C 2$, acetylcarnitine; $C 3$, propionylcarnitine; $C 4$, (iso)butyrylcarnitine; and $C 8: 1$, octenoylcarnitine.

Table 4. Analysis of urinary carnitine metabolites in infants receiving formulas enriched with $M C T$, children undergoing valproic acid therapy, and normal controls

\begin{tabular}{lccc}
\hline & $\begin{array}{c}\text { Subjects } \\
(n)\end{array}$ & $\begin{array}{c}\mathrm{C} 6, \mathrm{C} 8>\mathrm{MC} \\
\text { (positive) }\end{array}$ & $\begin{array}{c}\mathrm{C} 6, \mathrm{C} 8<\mathrm{MC} \\
\text { (negative) }\end{array}$ \\
\hline MCT feeding & 8 & 8 & 0 \\
VPA therapy* & 13 & 9 & 4 \\
Normal controls $\dagger$ & 25 & 0 & 25 \\
\hline
\end{tabular}

* VPA, valproic acid. Includes three subjects from Table 2.

$\dagger$ Includes 14 subjects from Table 2.
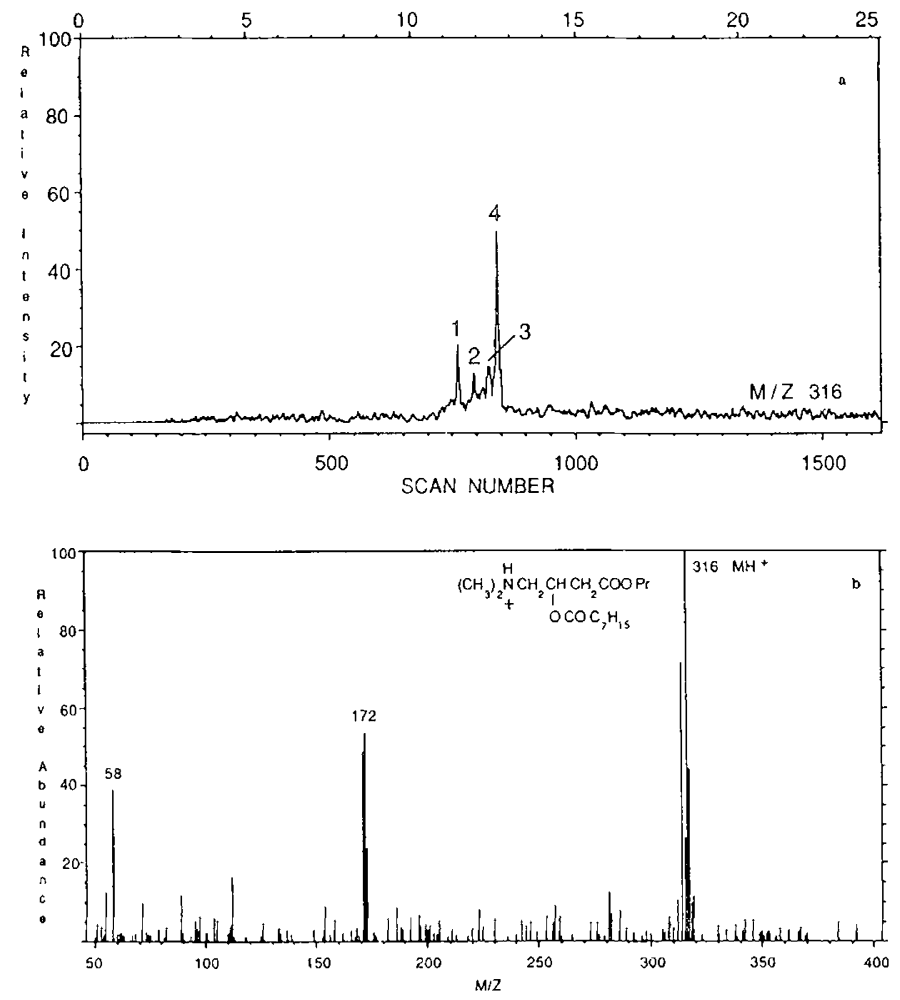

Fig. 4. $a$, Reconstructed mass chromatogram of $\mathrm{m} / \mathrm{z} 316\left(\mathrm{MH}^{+}\right.$for $\mathrm{N}$-demethylpropyl esters of saturated eight-carbon acylcarnitines) from the GC/MS analysis (17) of a urine specimen from a patient treated with valproic acid. Peak 1 represents valproylcarnitine, peak 2 represents an unidentified eight-carbon acylcarnitine, peak 3 is an impurity in the sample, and peak 4 represents C8. The identities of peaks 1 and 4 were confirmed by comparison with retention times and mass spectra of authentic standards. $b$, Chemical ionization (methane) mass spectrum of $\mathrm{N}$-demethylpropyl ester of $\mathrm{C} 8$ ( peak 4 in $a$ ).

Table 5. Quantitation of $C 6$ and $C 8$ in urine from patients with $M C A D$ deficiency, with and without L-carnitine therapy; from children receiving valproic acid; and from infants receiving formulas enriched with $M C T^{*}$

\begin{tabular}{lll}
\hline & \multicolumn{1}{c}{ C6 } & \multicolumn{1}{c}{ C8 } \\
\hline MCADD + carnitine & $1.07(0.04-14.3)$ & $6.10(0.42-134.8)$ \\
MCADD - carnitine & $0.08(0.03-0.82)$ & $0.38(0.10-4.21)$ \\
VPA & $0.19(0-2.10)$ & $0.71(0.09-14.6)$ \\
MCT & $0.31(0.07-1.06)$ & $0.42(0.07-0.69)$ \\
\hline
\end{tabular}

* Values are expressed in $\mathrm{mmol} / \mathrm{mol}$ creatinine and represent median (range) of each group of six patients. MCADD, MCAD deficiency; VPA, valproic acid.

able to demonstrate that MCAD deficiency can be reliably detected in the asymptomatic state without carnitine loading. However, the method is not entirely specific due to the excretion of significant amounts of $\mathrm{MC}$ in children with other inborn errors of fatty acid oxidation and in those receiving MCT or valproic acid. A history of such intake must be taken into account if the diagnosis of MCAD deficiency is considered through analysis of urinary acylcarnitines.

Acknowledgments. The authors thank Denise Alexander for her expert technical assistance; Dr. M. J. Bennett, Children's Hospital of Philadelphia, for his help with the GS/MS analysis of fatty acids; and Dr. L. L. Bieber, Michigan State University, for promoting collaboration and giving helpful advice. 


\section{REFERENCES}

1. Roe CR, Coates PM 1989 Acyl-CoA dehydrogenase deficiencies. In: Scriver CR, Beaudet AL, Sly WS, Valle D (eds) The Metabolic Basis of Inherited Disease. McGraw-Hill, New York, pp 889-914

2. Coates PM, Hale DE, Stanley CA, Corkey BE, Cortner JA 1985 Genetic deficiency of medium-chain acyl coenzyme A dehydrogenase: studies in cultured skin fibroblasts and peripheral mononuclear leukocytes. Pediatr Res 19:671-676

3. Amendt BA, Rhead WJ 1985 Catalytic defect of medium-chain acyl-coenzyme A dehydrogenase deficiency: lack of both cofactor responsiveness and biochemical heterogeneity in eight patients. J Clin Invest 76:963-968

4. Yokota I, Indo Y, Coates PM, Tanaka K 1990 Molecular basis of medium chain acyl-coenzyme A dehydrogenase deficiency. J Clin Invest 86:10001003

5. Rinaldo P, O'Shea JJ, Coates PM, Hale DE, Stanley CA, Tanaka K 1988 Medium-chain acyi-CoA dehydrogenase deficiency: diagnosis by stable isotope dilution measurement of urinary $n$-hexanoylglycine and 3-phenyl propionylglycine. N Engl J Med 319:1308-1313

6. Millington DS, Roe CR. Maltby DA 1984 Application of high resolution fast atom bombardment and constant $B / E$ ratio linked scanning to the identification and analysis of acylcarnitines in metabolic disease. Biomed Mass Spectrom 11:236-241

7. Rinaldo P, O'Shea JJ, Goodman SI, Miller LV, Fennessey PV, Whelan DT, Hill RE, Tanaka K 1989 Comparison of urinary acylglycines and acylcarnitines as diagnostic markers of medium-chain Acyl-CoA dehydrogenase deficiency. J Inherited Metab Dis 12:325-328

8. Millington DS, Roe CR 1989 Medium-chain acyl-CoA dehydrogenase deficiency. [letter] N Engl J Med 320:219

9. Millington DS, Norwood DL, Kodo N, Roe CR, Inoue F 1989 Application of fast atom bombardment with tandem mass spectrometry and liquid chromatography/mass spectrometry to the analysis of acylcarnitines in human urine, blood and tissue. Anal Biochem 180:331-339

10. Schmidt-Sommerfeld E, Penn D, Kerner J, Bieber LL, Rossi TM, Lebenthal E 1989 Quantitation of urinary carnitine esters in a patient with mediumchain acyl-coenzyme A dehydrogenase deficiency: effect of metabolic state and L-carnitine therapy. J Pediatr 115:577-582

11. Kerner J, Bieber LL 1985 Isolation and identification of methyloctanylcarnitines from human urine. Prep Biochem 15:237-257

12. Schmidt-Sommerfeld E, Penn D, Kerner J, Bieber LL 1989 Analysis of acylcarnitines in normal human urine with the radioisotopic exchange-high performance liquid chromatography (HPLC) method. Clin Chim Acta 181:231-238

13. Schmidt-Sommerfeld E, Penn D, Bieber LL, Kerner J, Rossi TM, Lebenthal E 1990 Carnitine ester excretion in pediatric patients receiving parenteral nutrition. Pediatr Res 28:158-165

14. Bieber LL, Kerner J 1986 Short-chain acylcarnitines identification and quantitation. Methods Enzymol 123:264-276

15. Kerner L, Bieber LL 1983 A radioisotopic exchange method for quantitation of short-chain acid-soluble acylcarnitines. Anal Biochem 134:459-466

16. Bohmer T, Bremer J 1986 Propionylcarnitine, physiological variations in vivo. Biochim Biophys Acta 152:559-567
17. Huang ZH, Gage DA, Bieber LL, Sweeley CC 1991 Analysis of acylcarnitines as their $\mathrm{N}$-demethylated ester derivatives by gas chromatography-chemical ionization mass spectrometry. Anal Biochem 199:89-105

18. Yokota I, Coates PM, Hale DE, Rinaldo P 1991 Molecular survey of a prevalent mutation, $985 \mathrm{~A}$-G-transition and identification of five infrequent mutations in the medium chain acyl-CoA dehydrogenase gene in 55 patients with medium chain acyl-COA dehydrogenase deficiency. Am J Hum Genet 49:1280-1291

19. Burlina AB, Zacchello F, Dioniso-Vici C, Bertini E, Sabetta G, Bennett MJ, Hale DE, Schmidt-Sommerfeld E, Rinaldo P 1991 A new clinical phenotype in three patients with biochemical evidence of a defect in branched-chain acyl-CoA oxidation. Lancet 338:1522-1523

20. Rinaldo P, Pierpont ME, Hale DE, Kolvraa S, Rhead WJ, Schmidt-Sommerfeld E, Gregersen N 1991 Clinical, biochemical and molecular heterogeneity in a family with medium chain acyl-CoA dehydrogenase deficiency. Am J Hum Genet 49(suppl):103(abstr)

21. Bieber LL 1988 Carnitine. Annu Rev Biochem 57:261-283

22. Millington DS, Bohan TP, Roe CR, Yergey AL, Liberato DJ 1985 Valproylcarnitine: a novel drug metabolite identified by fast atom bombardment and thermospray liquid chromatography-mass spectrometry. Clin Chim Acta 145:69-76

23. Duran M, Ketting D, Dorland L, Wadman SK 1985 The identification of acylcarnitines by desorption chemical ionization mass spectrometry. J Inherited Metab Dis 8[suppl 2]:143-144

24. Tracey CM, Chalmers RA, Rosankiewicz C, DeSousa C, Stacey TE 1986 Acylcarnitines in urine in medium-chain acyl-CoA dehydrogenase deficiency measured by quantitative high-pressure liquid chromatography. Biochem Soc Trans 14:700-701

25. Bhuiyan AKMJ, Watmough NJ, Turnbull DM, Aynsley-Green A, Leonard JV. Bartlett K 1987 A new simple screening method for the diagnosis of mediumchain acyl-CoA dehydrogenase deficiency. Clin Chim Acta 165:39-44

26. Montgomery JA, Mamer OA 1989 Measurement of urinary-free and acylcarnitines: quantitative acylcarnitine profinding in normal humans and in several patients with metabolic errors. Anal Biochem 176:85-95

27. Minkler PE, Ingalls ST, Hoppel CL 1990 High-performance liquid chromatographic separation of acylcarnitines following derivatisation with $4^{\prime}$-bromophenacyl trifluoromethanesulfonate. Anal Biochem 185:29-35

28. Penn D, Schmidt-Sommerfeld E 1990 Effect of medium-chain triglyceride administration upon carnitine and carnitine esters in the plasma, urine and bile of neonatal pigs. Pediatr Res 27:113A(abstr)

29. Ito M Ikeda Y. Arnez JG, Finocchiaro G Tanaka K 1990 The enzymatic basis for the metabolism and inhibitory effects of valproic acid: dehydrogenation of valproyl-CoA by 2-methyl-branched-acyl-CoA dehydrogenase. Biochim Biophys Acta 1034:213-218

30. Roe CR, Millington DS, Maltby DA, Kinnebrew P 1986 Recognition of medium-chain acyl-CoA dehydrogenase deficiency in asymptomatic siblings of children dying of sudden death or Reye-like syndromes. J Pediatr 108:1318

31. Amendt BA, Rhead WJ 1986 The multiple acyl-coenzyme A dehydrogenation disorders, glutaric aciduria type II and ethylmalonic-adipic aciduria. $\mathrm{J}$ Clin Invest 78:205-213

32. Loehr JP, Goodman SI, Frerman E i 990 Glutaric acidemia type II: heterogeneity of clinical and biochemical phenotypes. Pediatr Res 27:311-315 